\section{Tuft cells restrain PDAC progression}

Pancreatic tuft cells are produced by the potentially oncogenic process of acinar-to-ductal metaplasia (ADM), but what part these cells play in pancreatic tumorigenesis was largely unknown. A new study now suggests that tuft cells have a protective role in the progression of pancreatic ductal adenocarcinoma (PDAC).

Chemosensory tuft cells are found throughout the digestive tract, with limited functional data indicating multifaceted roles in sensing and responding to inflammatory conditions. Previous work by author Kathleen DelGiorno found that tuft cells form in the pancreas by ADM, undetermined. ADM and its role in pancreatic cancer was a focus of research by author Geoffrey Wahl, so a collaborative study was formed.

The researchers used several methods to determine the functional role of tuft cells in pancreatic tumorigenesis. "We used genetically but their physiological effects were engineered mouse models lacking tuft cells to study how pancreatic tumours form in their absence, and we used single-cell sequencing and histopathology to evaluate the effects of tuft cell deletion on the neoplastic stroma," says DelGiorno. The investigators found that tuft cell ablation in mouse models of PDAC accelerated tumorigenesis.

"Pancreatic tuft cells act to restrain tumorigenesis rather than seed or drive cancer formation," explains DelGiorno.

Using small cell number RNA-sequencing and metabolic profiling to examine tuft cell mechanisms, a role for eicosanoid secretion in curbing activation of the tumour stoma was highlighted. "While some eicosanoids have been described to be pro-tumorigenic, we found that pancreatic neoplasia exhibit high levels of anti-tumorigenic prostaglandin D2 to which tuft cells substantially contribute," reports Wahl, adding that tuft cells might tuft cell ablation in mouse models of PDAC accelerated tumorigenesis
Credit: Scanning electron microscopy image of a tuft cell. Image courtesy of G. Wahl and K. DelGiorno.

also produce other paracrine factors that modulate tumour progression.

Finally, the investigators also examined tissue samples from patients with PDAC $(n=197)$ and its precursor lesions $(n=45)$. They found gene expression signatures associated with tuft cells were higher in precursor lesions than PDAC, supporting the hypothesis that tuft cells suppress disease progression.

"In the course of these studies we identified a number of other potential tuft cell mechanisms of disease suppression worth evaluating," says DelGiorno. "We are also interested in understanding how conditions known to predispose to pancreatic and other cancers, such as obesity and inflammation, induce cell state-switching events," adds Wahl.

Iain Dickson

ORIGINAL ARTICLE DelGiorno, K. E. et al. Tuft cells inhibit pancreatic tumorigenesis in mice by producing prostaglandin D2. Gastroenterology https://doi.org/10.1053/j.gastro.2020.07.037 (2020)

BARRETT OESOPHAGUS

\title{
Detecting Barrett oesophagus using Cytosponge
}

Improved detection of Barrett oesophagus can help prevent progression to oesophageal adenocarcinoma. Now, a trial of a non-endoscopic test, Cytosponge-trefoil factor 3 (TFF3), has demonstrated improved detection of Barrett oesophagus compared with standard management.

Cytosponge-TFF3 is a device that can be used in the primary care setting. The device is swallowed and collects oesophageal cells, and an in vitro test for the biomarker TFF3 identifies intestinal metaplasia. "This is a new test and before this there had been no randomized trial data out there to show that this approach can diagnose more cases of Barrett oesophagus and early cancer," says author Rebecca Fitzgerald.

The randomized controlled trial was conducted in 109 general practice clinics in England. Eligible participants (those aged 50 years or older and who had been prescribed acid-suppressant therapy for at least 6 months in the previous year) were randomly assigned to the intervention group ( $n=6,983)$, who were offered the CytospongeTFF3 procedure, or the control group ( $n=6,531$ ), who instead had an endoscopy if referred by the general practitioner. After accounting for those who did not meet all eligibility criteria or withdrew from the study, there were 6,834 in the intervention group and 6,388 in the control group.

Of 1,654 participants who successfully swallowed the Cytosponge device, 221 (13\%) tested positive for TFF3 and underwent endoscopy, with 131 (8\%) being diagnosed with Barrett oesophagus or cancer. In the intervention group and control group, 140 (2\%) participants and $13(<1 \%)$ participants, respectively, were diagnosed with Barrett oesophagus during an average of 12 months follow-up (overall rate ratio 10.2 (95\% Cl 5.8-18.1) and $10.6(95 \% \mathrm{Cl}$ 6.0-18.8; $P<0.0001)$ when adjusted for cluster randomization).

Thus, offering Cytosponge-TFF3 increased the diagnosis of Barrett oesophagus by tenfold compared with usual medication and endoscopy referral as determined by the clinician. The next steps for clinical implementation include evaluating the health economics, determining how Cytosponge-TFF3 fits into clinical care pathways, and investigating additional biomarkers for surveillance, according to Fitzgerald. "The COVID-19 situation is accelerating adoption of this technology as it is less aerosol-generating than endoscopy and can be performed by a single member of staff in an office setting outside of the hospital."

Jordan Hindson with standard management
ORIGINAL ARTICLE Fitzgerald, R. et al.

Cytosponge-trefoil factor 3 versus usual care to identify Barrett's oesophagus in a primary care setting: a multicentre, pragmatic, randomised controlled trial. Lancet 396, 333-344 (2020) 